\title{
The effect of neutropenia on the clinical pharmacokinetics of vancomycin in adults
}

\author{
Didi Bury ${ }^{1} \cdot$ Rob ter Heine ${ }^{2}$ (D) Ewoudt M. W. van de Garde ${ }^{3,4} \cdot$ Marten R. Nijziel $^{5} \cdot$ Rene J. Grouls $^{1} \cdot$ \\ Maarten J. Deenen ${ }^{1,6}$
}

Received: 23 August 2018 / Accepted: 26 February 2019 / Published online: 15 March 2019

(C) The Author(s) 2019

\begin{abstract}
Aim There is accumulating evidence that neutropenic patients require higher dosages of vancomycin. To prevent sub-therapeutic drug exposure, it is of utmost importance to obtain adequate exposure from the first dose onwards. We aimed to quantify the effect of neutropenia on the pharmacokinetics of vancomycin.

Methods Data were extracted from a matched patient cohort of patients known with (1) hematological disease, (2) solid malignancy, and (3) patients not known with cancer. Pharmacokinetic analysis was performed using non-linear mixed effects modeling with neutropenia investigated as a binary covariate on clearance and volume of distribution of vancomycin.

Results A total of 116 patients were included (39 hematologic patients, 39 solid tumor patients, and 38 patients not known with cancer). In total, 742 paired time-concentration observations were available for the pharmacokinetic analysis. Presence of neutropenia showed to significantly $(p=0.00157)$ increase the clearance of vancomycin by $27.7 \%$ (95\% CI $10.2-46.2 \%$ ), whereas it did not impact the volume of distribution $(p=0.704)$.

Conclusions This study shows that vancomycin clearance is increased in patients with neutropenia by $27.7 \%$. Therefore, the vancomycin maintenance dose should be pragmatically increased by $25 \%$ in neutropenic patients at the start of treatment. Since the volume of distribution appeared unaffected, no adjustment in loading dose is required. These dose adjustments do not rule out the necessity of further dose individualization by means of therapeutic drug monitoring.
\end{abstract}

Keywords Vancomycin $\cdot$ Pharmacokinetics $\cdot$ Neutropenia $\cdot$ NONMEM $\cdot$ Hematology

Electronic supplementary material The online version of this article (https://doi.org/10.1007/s00228-019-02657-6) contains supplementary material, which is available to authorized users.

Rob ter Heine

r.terheine@ radboudumc.nl

1 Department of Clinical Pharmacy, Catharina Hospital Eindhoven, Michelangelolaan 2, 5623 EJ Eindhoven, The Netherlands

2 Department of Pharmacy, Radboud University Medical Center, Geert Grooteplein Zuid 10, 6525 GA Nijmegen, The Netherlands

3 Department of Clinical Pharmacy, St. Antonius Hospital, Koekoekslaan 1, 3435 CM Nieuwegein, The Netherlands

4 Division of Pharmacoepidemiology and Clinical Pharmacology, Department of Pharmaceutical Sciences, Faculty of Science, Utrecht University, Universiteitsweg 99, 3584 CG Utrecht, The Netherlands

5 Department of Haematology, Catharina Hospital Eindhoven, Michelangelolaan 2, 5623 EJ Eindhoven, The Netherlands

6 Department of Clinical Pharmacy \& Toxicology, Leiden University Medical Center, Albinusdreef 2, 2333 ZA Leiden, The Netherlands

\section{Introduction}

Vancomycin is an effective antibiotic agent with antimicrobial activity against infections caused by gram-positive bacteria, including methicillin-resistant Staphylococcus aureus. Vancomycin is frequently used to treat potential infections in febrile neutropenic patients with hematological disease $[1,2]$. For an effective treatment, it is important to quickly reach therapeutic plasma concentrations of vancomycin that is guided using therapeutic drug monitoring. Current international guidelines recommend a target range for the trough concentration of $15-20 \mathrm{mg} / \mathrm{L}$ for intermittent infusion schedules, which in daily practice can be achieved with a vancomycin dose of $15-20 \mathrm{mg} / \mathrm{kg}$ given every $8-12 \mathrm{~h}$ in most patients with normal renal function [3]. Interestingly, patients with hematological malignancies appear to require much higher dosages of vancomycin than patients not known with malignant disease in order to achieve therapeutic plasma concentrations [4, 5]. 
This difference is likely due to an increased clearance of vancomycin in this specific patient population. Specifically, recent studies show that especially neutropenia is a risk factor associated with the increased clearance of vancomycin [6-8].

Patients with hematological malignancies are often neutropenic due to the disease or during and after chemotherapeutic treatment of the disease. Febrile neutropenia is strongly associated with an increased mortality risk with a mortality rate in severe septic patients of up to $40-50 \%[9,10]$. Furthermore, it is associated with increased health care costs $[11,12]$. Thereby, it is of utmost importance to obtain adequate antibiotic drug exposure from the first dose onwards, with as little as dose titration necessary, especially in this vulnerable group of patients. Using the current standard vancomycin dosingdosage regimen, therapeutic plasma concentrations are often reached late in neutropenic patients with hematological malignancies, as dose requirements are often higher than initially prescribed. Only a minority of hematologic cancer patients reaches therapeutic plasma concentrations following standard dosingdosage regimens [4, 5, 13-15]. Nonetheless, despite this knowledge of the augmented clearance and necessity for higher vancomycin dosages, the effect of neutropenia on the clearance of vancomycin has thus far not yet properly been quantified, hampering a proper dose advice. Quantification of the effect of neutropenia on the pharmacokinetics of vancomycin allows the development of an effective dosage regimen for this specific patient population. However, in most studies thus far conducted, hematological disease and/ or cancer-and not specifically neutropenia - was taken as covariate for estimation of the altered vancomycin pharmacokinetics. As a result, the available effect size estimations on clearance and volume of distribution of vancomycin are not consistent and vary widely among studies. Therefore, the aim of this study was to properly quantify the effect of neutropenia on the pharmacokinetics of vancomycin using non-linear mixed effect modeling.

\section{Patients and methods}

\section{Study design and patient population}

The primary objective of the study was to quantify the effect of neutropenia on the pharmacokinetics of vancomycin. To be able to compare the pharmacokinetics of vancomycin between various patient populations, a retrospective, matched cohort design was used consisting of three cohorts of patients, i.e., patients with (1) hematologicaldisease, (2) solid tumor, and (3) patients not known with cancer. The flowchart of the inclusion of patients is provided in Fig. 1. Patients were matched on age, gender, and year of treatment. Inclusion criteria were age 18 years or older, treatment with intravenous vancomycin therapy for $\geq$ 2 days due to bacteriologically documented or suspected gram-positive infections, plus at least one available vancomycin plasma concentration. Patients who had received a single dose of vancomycin and patients on dialysis were excluded. Patient and treatment characteristics were retrospectively collected by analysis of the Electronic Healthcare Record System and by the use of the Santeon Farmadatabase. The study was approved by the medical ethical committee of the Catharina Hospital Eindhoven and was conducted following the principles of the Declaration of Helsinki.

\section{Patient and treatment characteristics}

The following baseline patient and treatment characteristics were obtained: underlying malignant diagnosis, age, gender, height, total body weight (TBW), aspartate aminotransferase (AST), alanine amino transferase (ALT), serum creatinine, white blood cell count (WBC), absolute neutrophil count, C-reactive protein (CRP), albumin, and type of chemotherapy. Creatinine clearance was calculated according to the Cockcroft-Gault formula [16]. All variables were registered as the last known value within 7 days before the start of the vancomycin therapy; for the ANC, values within 2 days of the start of treatment were also accepted in case no baseline values were available. Except for albumin, and CRP, for which a time window of 2 weeks and 3 days was maintained, respectively. Current chemotherapy was registered as yes or no for the malignancy groups. During treatment with vancomycin, all available data on serum creatinine, white blood cell count, absolute neutrophil count, and Creactive protein were extracted. Furthermore, loading dose, daily dose, infusion time, peak serum levels, trough serum levels, frequency of dosing, dosing interval, dose adjustments, frequency of dose adjustments, duration of vancomycin therapy, and time of blood sampling were extracted.

\section{Bioanalysis}

Plasma concentrations of vancomycin were measured during routine clinical care on a Viva-E system, using a validated spectrophotometric homogeneous enzyme immunoassay (Emit@ 2000, Siemens Healthcare Diagnostics). The calibration curve ranged from 0 to $50 \mathrm{mg} / \mathrm{L}$. Besides a calibration curve, quality control samples of known concentrations (7.4, 17.5 , and $34.0 \mathrm{mg} / \mathrm{L}$ ) were incorporated in each run with coefficients of variation of $4.2 \%, 2.4 \%$, and $4.6 \%$, respectively. Peak plasma concentrations were collected within 30-60 min after the end of infusion, and trough concentrations immediately before the next dose.

\section{Pharmacokinetic analysis}

Pharmacokinetic model development Pharmacokinetic analysis was performed by means of non-linear mixed effects modeling, the software package NONMEM V7.3, with 


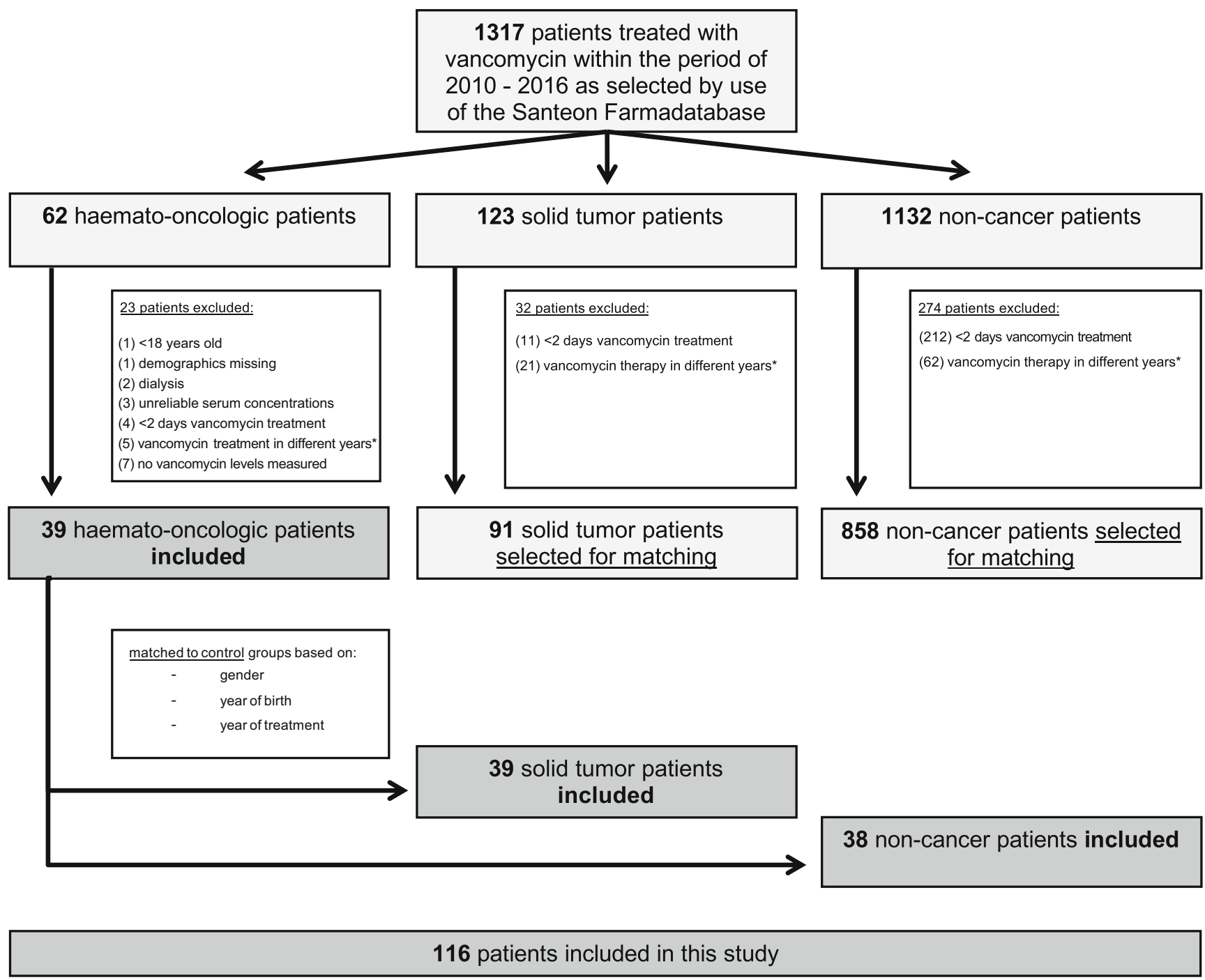

Fig. 1 Flowchart of the study

Piraña v2.9.4 as an interface for NONMEM, R statistics v3.4.0 and Perl Speaks Nonmem [17]. Imprecision of parameter estimates was calculated with the sampling importance resampling procedure [18]. Model development was guided by physiological plausibility and goodness-of-fit, as assessed with standard goodness-of-fit plots and prediction-corrected visual predictive checks [19]. Non-nested models were assessed with the Akaike Information Criterion [20].

The previously developed pharmacokinetic model for vancomycin by Staatz et al. was used as a starting point of our analysis [21]. This model described vancomycin pharmacokinetics with distribution in one compartment of which the volume was linearly scaled to total body weight. Clearance of vancomycin was linearly related to creatinine clearance, as estimated with the Cockcroft-Gault formula. Lastly, residual error was described with a combined additive and proportional error, and inter-individual variability was described with an exponential error. After initial fitting of the model, we investigated the necessity of model adjustments to better describe our population. Furthermore, as vancomycin is a known hydrophilic drug [22], scaling of the volumes of distribution to total body weight may not be optimal, as it is unlikely that adipose tissue evenly contributes to distribution vancomycin as non-adipose tissue. We, therefore, investigated the performance scaling of the volume of distribution to normal fat mass and fat-free mass, as proposed recently [23]. When scaling to fat-free mass, we scaled the pharmacokinetic parameters to a mass of $57.2 \mathrm{~kg}$, corresponding to the fat-free mass of a typical man of $1.80 \mathrm{~m}$ and a total body weight of $70 \mathrm{~kg}$.

A posteriori power and covariate analysis Neutropenia was tested as a covariate for the pharmacokinetics of vancomycin. Presence of neutropenia was defined as absolute neutrophil count $(\mathrm{ANC})<1.5$ cells $/ \mathrm{nL}$. Absence of neutropenia was defined as $\mathrm{ANC} \geq 1.5$ cells $/ \mathrm{nL}$, or in case no differential of the WBC was available, a normal WBC value defined as WBC > 
4 cells/nL plus no clinical suspicion of neutropenia. During covariate analysis, statistical significance was calculated from the decrease in objective function. A decrease of 3.84 points or more, associated with a $p$ value of $<0.05$, was considered statistically significant. Non-nested models were assessed with the Akaike Information Criterion. [20] After the development of the base model, we investigated the power in to detect a clinically relevant (defined as a $>33.3 \%$ ) change in clearance or total volume of distribution because of neutropenia at a significance level of $5 \%$. For this purpose, we performed a stochastic simulation and estimation study, using the SSE tool in Perl Speaks Nonmem [17] with 500 virtual studies for each power calculation. After the power analysis, we investigated the impact of neutropenia as binary covariate on clearance and total volume of distribution.

Simulation study With the final model, we performed a simulation of the pharmacokinetics of vancomycin in an intermittent and continuous dosingdosage regimen of $1800 \mathrm{mg}$ per $24 \mathrm{~h}$ in a three times daily intermittent or continuous regimen. This dose was based on a previously developed and validated nomogram [24]. Alternate dosing schemes were explored at the discretion of the investigators to overcome subtherapeutic exposure.

\section{Results}

Patient characteristics Within the period of 2010-2016, a total of 116 matched patients were enrolled in the study, of which 39 patients with a hematologic malignancy, 39 patients with a solid tumor, and 38 patients not known with cancer. Table 1 shows the patient baseline characteristics and Table 2 shows the underlying malignancies of the oncologic patients. Primary diagnoses of the patients with a hematological malignancy was acute myeloid leukemia (59\%), Hodgkin's or nonHodgkin's lymphoma (21\%), or other types of leukemia (21\%), of which in total $80 \%$ of the patients were on current chemotherapy schedules. Primary diagnoses of the patients with solid tumors were gastro-intestinal cancer (72\%), urogenital cancer (26\%), and CNS tumor (3\%), of which in total $33 \%$ of the patients were on current chemotherapeutic treatment regimens. In a total of $5756(4948 \%)$ patients, ANC values were known within the specified time frame of 7 days prior to treatment or within 2 days after the start of treatment. In another $10(9 \%)$ patients, the WBC was too low $(\leq 0.10$ cells $/ \mathrm{nL}$ ) in order to determine a differential count, and they could, therefore, be categorized as neutropenic based on their low WBC values. In the remaining 4950 (4243\%) patients, no differential WBC was available. However, in all these patients, the WBC exceeded the minimum value of 4 cells $/ \mathrm{nL}$. In addition, no neutropenia was suspected from their clinical presentation and these patients were, therefore, categorized as non-neutropenic. In total, this resulted in a total of 31 neutropenic patients, of which 29 were known with hematological disease. The median time window between determination of ANC or white blood cell count and the start of vancomycin treatment was 0 days (range $0-7$ days).

\section{Pharmacokinetic analysis}

Pharmacokinetic model development After initial fitting of the model, we observed under-prediction of vancomycin plasma concentrations at the end of the dosing interval, indicating the presence of a peripheral distribution compartment. Estimation of a peripheral compartment significantly $(p<0.001)$ improved model fit, and, therefore, this was retained in the model. Furthermore, when investigating the impact of body size on the volume of distribution, fat tissue did not contribute to the distribution of vancomycin (data not shown) and, therefore, volume of distribution was allometrically scaled to fat-free mass. This was associated with a better model fit compared to scaling to total body weight, observed as decrease of the Akaike Information Criterion of $>10$ points. For the same reason, the intercompartmental clearance between the central and peripheral compartment was allometrically scaled to fat-free mass. Because clearance of vancomycin was already related to body weight, with body weight being one of the variables in the Cockcroft-Gault creatinine clearance equation, clearance was not additionally scaled to body size for the base model. The detailed modeling results and parameter estimates for the base and final model are shown in the supplementary files of this manuscript.

A posteriori power and covariate analysis We found that in our population, when using the developed base model, we had $93.8 \%$ and $83.4 \%$ power to detect a respective change of $33.3 \%$ in clearance and total volume due to neutropenia. When investigating the presence of neutropenia as a covariate for clearance of vancomycin, we found that neutropenia relevantly and significantly $(p=0.00157)$ increased clearance in neutropenic patients by $27.7 \%$ (95\% confidence interval $10.2-46.2 \%)$. The presence of neutropenia did not significantly impact the volume of distribution $(p=0.704)$ and was, therefore, not included in the final model.

Simulation study To demonstrate the change in vancomycin levels between the current dosingdosage regimen and the algorithm obtained with our model, a simulation of the pharmacokinetics of vancomycin was performed for a typical male patient $(70 \mathrm{~kg}, 1.80 \mathrm{~m})$ with a normal kidney function (creatinine clearance of $104 \mathrm{ml} / \mathrm{min}$ ). Following continuous infusion of $1800 \mathrm{mg}$ vancomycin therapeutic levels were reached, while a neutropenic patient on the same dose reached subtherapeutic 
Table 1 Patient characteristics

\begin{tabular}{|c|c|c|c|c|c|}
\hline Characteristic & Total $(n=116)$ & Hematological malignancy $(n=39)$ & Solid tumor $(n=39)$ & Non-cancer $(n=38)$ & $s P$ \\
\hline \multicolumn{5}{|l|}{ Sex, $N(\%)$} & \multirow[t]{3}{*}{0.97} \\
\hline Male & $67(58 \%)$ & $22(56 \%)$ & $23(59 \%)$ & $22(58 \%)$ & \\
\hline Female & $49(42 \%)$ & $17(44 \%)$ & $16(41 \%)$ & $16(42 \%)$ & \\
\hline \multicolumn{4}{|l|}{ Age } & $60.1 \pm 13.4$ & 0.23 \\
\hline BMI & $25.5(5.9)$ & $24.0(6.2)$ & $26.9(6.2)$ & $26.3(7.2)$ & 0.002 \\
\hline \multicolumn{4}{|l|}{ WBC (cells/nL) } & $11.7(8.6)$ & $<0.001$ \\
\hline \multicolumn{4}{|l|}{ ANC $(\text { cells } / \mathrm{nL})^{\#}$} & $11.0(4.4)$ & $<0.001$ \\
\hline AST (U/L) & $28.0(29.0)$ & $25.5(35.5)$ & $34.0(30.5)$ & $29.5(35.5)$ & 0.06 \\
\hline $\operatorname{ALT}(\mathrm{U} / \mathrm{L})$ & $28.5(34.0)$ & $23.0(29.5)$ & $28.0(29.5)$ & $34.0(34.0)$ & 0.20 \\
\hline \multicolumn{4}{|l|}{$\begin{array}{l}\text { Albumin }(\mathrm{g} / \mathrm{L}) \\
\text { median }(\mathrm{IQR})\end{array}$} & $32.0(13.0)$ & 0.001 \\
\hline \multicolumn{5}{|c|}{ ln Cockcroft-Gault clearance (ml/min) } & 0.07 \\
\hline \multicolumn{5}{|c|}{ C-reactive protein $(\mathrm{mg} / \mathrm{L})$} & 0.07 \\
\hline
\end{tabular}

\# In 10 patients, no differential was performed due to low $\mathrm{WBC}(\mathrm{WBC} \leq 0.1$ cells $/ \mathrm{nL})$ and are therefore not included in the calculation of the median and IQR

$A L T$, alanine aminotransferase; $A N C$, absolute neutrophile count; $A S T$, aspartate aminotransferase; $B M I$, body mass index; $I Q R$, interquartile range; ln, natural logarithm; $W B C$, white blood cell count

levels. Increasing the vancomycin dose by $25 \%$ showed therapeutic levels in the same neutropenic patient. Plots of the vancomycin levels after continuous infusion in non-neutropenic and neutropenic patients are shown in Fig. 2 (panel a).

A similar simulation was performed for an intermittent infusion of vancomycin seen in Fig. 2 (panel b). With a dose of $1800 \mathrm{mg}$ vancomycin divided over three administrations resulted in subtherapeutic trough levels in the neutropenic patient. The non-neutropenic patient showed levels within this therapeutic range at the same dose. The vancomycin levels were comparable to the trough levels of the non-neutropenic patient after increasing the vancomycin dose by $25 \%$.

\section{Discussion}

The results of this study show that neutropenia is significantly associated with a $27.7 \%$ higher clearance of vancomycin. Thereby, as the systemic exposure is directly proportional to drug dose, the vancomycin maintenance dose should be pragmatically increased by $25 \%$ at the start of therapy in neutropenic patients with hematological diseases, followed by further therapeutic drug monitoring. Because in our study, neutropenia appeared not to affect volume of distribution; no increase in the loading dose seems required. Increasing the vancomycin maintenance dose immediately at the start of therapy

Table 2 Underlying malignancy

\begin{tabular}{lll}
\hline & Hematological malignancy $(n=39)$ & Solid tumor $(n=39)$ \\
\hline Underlying malignancy $(N)^{*}$ & AML (23) & Gastrointestinal cancer (28) \\
& HL /NHL (8) & Urogenital cancer (7) \\
& ALL (2) & Breast cancer (3) \\
& Other (6) & CNS malignancy (1) \\
Chemotherapy & & \\
Yes (\%) & $80 \%$ & $33 \%$ \\
No (\%) & $20 \%$ & $67 \%$ \\
\hline
\end{tabular}

$A L L$, acute lymphoblastic leukemia; $A M L$, acute myelocytic leukemia; HL/NHL, Hodgkin's/hon-Hodgkin's lymphoma 
Fig. 2 Simulation study results. Panel a Comparison of vancomycin levels after continuous infusion in a nonneutropenic patient $(1800 \mathrm{mg}$ day) and in a neutropenic patient $(1800 \mathrm{mg} /$ day and $2250 \mathrm{mg} /$ day $)$.

Panel b Comparison of vancomycin levels after intermittent infusion in a nonneutropenic patient with a dosage of $1800 \mathrm{mg}$ /day (600 mg TID*) and in a neutropenic patient with a dosage of $1800 \mathrm{mg} /$ day $(600 \mathrm{mg}$ TID) and $2250 \mathrm{mg} /$ day (750 mg TID). The shaded areas represent the therapeutic ranges. *TID, three times daily

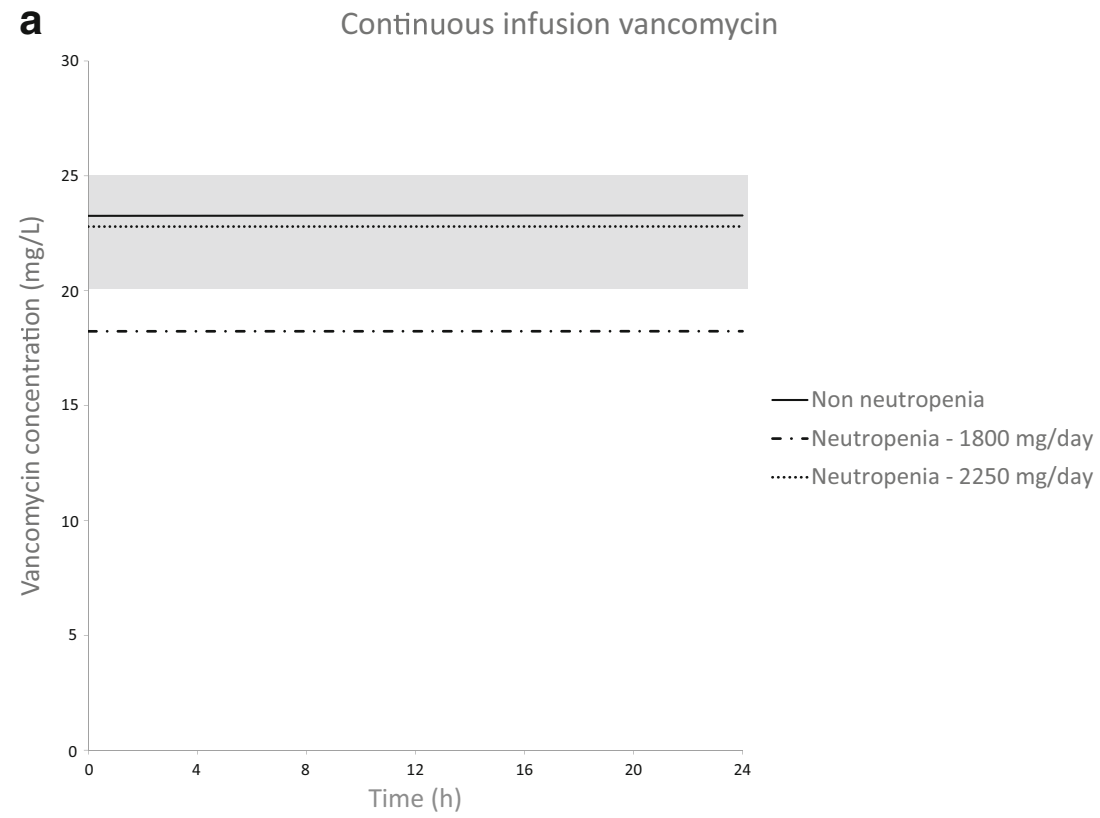

b Intermittent infusion vancomycin

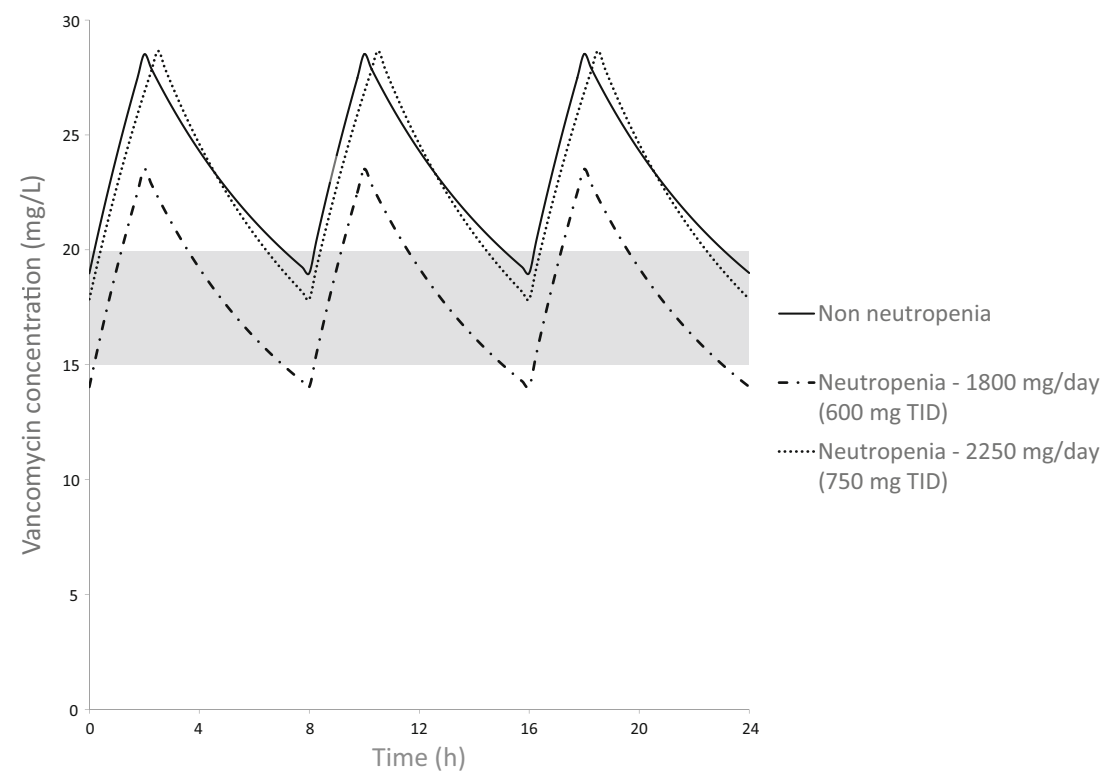

may prevent unnecessary subtherapeutic drug exposure, less dose titration after initiation of therapy, and may, thereby, increase effectiveness of treatment. This is of special importance in neutropenic patients with hematological disease, as these patients are often immunocompromised, and $S$. aureus infections are associated with high mortality rates of up to $40-50 \%$ $[9,10]$.

Our findings confirm the earlier exploratory study by Haeseker et al. who also revealed neutropenia as principal independent predictor of clearance as well as the study by Choi et al. who showed the association between neutropenia and subtherapeutic levels of vancomycin [6,7]. Similarly, Fernandez de Gatta et al. and Normand et al. found an increased clearance in neutropenic patients [4, 15]. Noteworthy, our study took into account all multiple peak and trough concentrations during the complete course of treatment with vancomycin. This is contrary to the study of Le Normand et al., where multiple blood samples were analyzed around a single vancomycin administration and Fernandez de Gatta et al. $[4,15]$, who measured serum vancomycin concentration only after the initial dose and at steady state. The contribution of our study to current literature is the development of a pharmacokinetic model of vancomycin in neutropenic and non-neutropenic patients. This allowed quantitative assessment of the effect of neutropenia on vancomycin clearance. Furthermore, in clinical practice, this model can serve as 
a useful tool to guide vancomycin dose individualization by means of therapeutic drug monitoring in both patient groups.

This study may have been limited by its retrospective nature, with potential variation in exact administration times of vancomycin and timing of blood collection for determination of vancomycin peak and trough levels. However, on the other hand, it may be considered a strength being a real-world study in a daily patient population. Although the influence of neutropenia is evident, the underlying mechanism of augmented clearance in neutropenic patients is still not completely understood. It is therefore an interesting topic for future research.

Physiological processes that are altered during febrile illness may play a role in changed pharmacokinetics. Renal hyperfiltration is considered as a possible mechanism to explain the reduced vancomycin levels in critically ill patients. This phenomenon might develop from a hyperdynamic circulation as a response to systemic inflammation and is associated with elevated renal perfusion $[25,26]$. However, as calculated creatinine clearance was an inherent part of the pharmacokinetic model, we postulate that in our analysis, potential hyperfiltration is already accounted for. Another hypothesis may be that serum albumin concentrations affect the observed vancomycin pharmacokinetics. Vancomycin is known to bind to serum albumin [27] and, therefore, decreased serum albumin concentrations may result in lower total (bound and unbound) vancomycin concentrations. Notably, serum albumin concentrations in the patients with hematological malignancies, i.e., most patients with neutropenia, were not different in comparison to non-cancer patients, as presented in Table 1 (off note, the significant difference in albumin levels in Table 1 is driven by the lower albumin levels in solid tumor patients). Therefore, variations in serum albumin concentrations seem an unlikely cause for the augmented clearance of vancomycin. Therefore, until the mechanism is unraveled, neutropenia should be considered a risk factor for decreased vancomycin exposure.

This study was not designed to investigate the effect of a higher maintenance dosage on mortality, costs, and overall outcome. In order to study the effect of the adjusted dosage regimen on outcome, a prospective study in hematologic patients with neutropenia comparing the standard maintenance dosage with a $25 \%$ higher dosage is necessary. Increased plasma concentrations of vancomycin are associated with increased risk of nephrotoxicity [3]. However, increasing the maintenance dose in neutropenic patients by $25 \%$ to reach therapeutic plasma concentrations is not likely to increase the risk of nephrotoxicity, as the data show that thereby no supratherapeutic - but therapeutic - plasma concentrations will be reached.

Lastly, it is unknown whether our results may be extrapolated to all neutropenic patients, as the majority of the neutropenia cases were in patients with hematological malignancy. Further studies in a more diverse patient group may help to answer this question.
To conclude, this study shows that neutropenia is associated with increased clearance of vancomycin. We suggest to pragmatically increase the initial vancomycin dose by $25 \%$ in this vulnerable patient population with neutropenia to increase the likelihood of pharmacokinetic target attainment, along with therapeutic drug monitoring to further tailor the dose of vancomycin.

\section{Compliance with ethical standards}

Transparency declarations None to declare. DB and MD had full access to all the data in the study and take responsibility for the integrity of the data and the accuracy of the data analysis. The specific contributions of each author are as follows:

Study concept and design: all authors

Acquisition, analysis, and interpretation of the data: DB, RtH, EvG, and MD

Drafting of the manuscript: DB, RtH, and MD

Critical revision of the manuscript: EvdG, MN, and RG.

Approval of the submitted and final version: all authors

Open Access This article is distributed under the terms of the Creative Commons Attribution 4.0 International License (http:// creativecommons.org/licenses/by/4.0/), which permits unrestricted use, distribution, and reproduction in any medium, provided you give appropriate credit to the original author(s) and the source, provide a link to the Creative Commons license, and indicate if changes were made.

\section{References}

1. Blennow O, Ljungman $\mathrm{P}$ (2016) The challenge of antibiotic resistance in haematology patients. Br J Haematol 172:497-511. https:// doi.org/10.1111/bjh.13816

2. Freifeld AG, Bow EJ, Sepkowitz KA, Boeckh MJ, Ito JI, Mullen CA, Raad II, Rolston KV, Young JAH, Wingard JR (2011) Clinical practice guideline for the use of antimicrobial agents in neutropenic patients with cancer: 2010 update by the infectious diseases society of America. Clin Infect Dis 52:e56-e93. https://doi.org/10.1093/ $\mathrm{cid} / \mathrm{cir} 073$

3. Rybak M, Lomaestro B, Rotschafer JC, Moellering R Jr, Craig W, Billeter M, Dalovisio JR, Levine DP (2009) Therapeutic monitoring of vancomycin in adult patients: a consensus review of the American Society of Health-System Pharmacists, the Infectious Diseases Society of America, and the Society of Infectious Diseases Pharmacists. Am J Health Syst Pharm 66:82-98. https:// doi.org/10.2146/ajhp080434

4. Fernandez de Gatta MM, Fruns I, Hernandez JM et al (1993) Vancomycin pharmacokinetics and dosage requirements in hematologic malignancies. Clin Pharm 12:515-520

5. Hochart C, Berthon C, Corm S, Gay J, Cliquennois M, Tricot S, Alfandari $S$ (2011) Vancomycin serum concentration during febrile neutropenia in patients with acute myeloid leukemia. Med Mal Infect 41:652-656. https://doi.org/10.1016/j.medmal.2011.09.014

6. Haeseker MB, Croes S, Neef C, Bruggeman CA, Stolk LML, Verbon A (2014) Vancomycin dosing in neutropenic patients. PLoS One 9:e112008. https://doi.org/10.1371/journal.pone. 0112008

7. Choi MH, Choe YH, Lee S-G, Jeong SH, Kim JH (2017) Neutropenia is independently associated with sub-therapeutic 
serum concentration of vancomycin. Clin Chim Acta 465:106-111. https://doi.org/10.1016/j.cca.2016.12.021

8. Hirai K, Ishii H, Shimoshikiryo T, Shimomura T, Tsuji D, Inoue K, Kadoiri T, Itoh K (2016) Augmented renal clearance in patients with febrile neutropenia is associated with increased risk for subtherapeutic concentrations of vancomycin. Ther Drug Monit 38: 706-710. https://doi.org/10.1097/FTD.0000000000000346

9. Legrand M, Max A, Peigne V, Mariotte E, Canet E, Debrumetz A, Lemiale V, Seguin A, Darmon M, Schlemmer B, Azoulay É (2012) Survival in neutropenic patients with severe sepsis or septic shock. Crit Care Med 40:43-49. https://doi.org/10.1097/CCM. 0b013e31822b50c2

10. Lyman GH, Michels SL, Reynolds MW, Barron R, Tomic KS, Yu J (2010) Risk of mortality in patients with cancer who experience febrile neutropenia. Cancer 116:5555-5563. https://doi.org/10. $1002 /$ cncr.25332

11. Liou SY, Stephens JM, Carpiuc KT, Feng W, Botteman MF, Hay JW (2007) Economic burden of haematological adverse effects in cancer patients: a systematic review. Clin Drug Investig 27:381396

12. Michels SL, Barron RL, Reynolds MW, Smoyer Tomic K, Yu J, Lyman GH (2012) Costs associated with febrile neutropenia in the US. Pharmacoeconomics 30:809-823. https://doi.org/10.2165/ 11592980-000000000-00000

13. Al-Kofide H, Zaghloul I, Al-Naim L (2010) Pharmacokinetics of vancomycin in adult cancer patients. J Oncol Pharm Pract 16:245250. https://doi.org/10.1177/1078155209355847

14. Buelga DS, del Mar Fernandez de Gatta M, Herrera EV et al (2005) Population pharmacokinetic analysis of vancomycin in patients with hematological malignancies. Antimicrob Agents Chemother 49: 4934 4941. https://doi.org/10.1128/AAC.49.12.4934-4941.2005

15. Le Normand Y, Milpied N, Kergueris MF, Harousseau JL (1994) Pharmacokinetic parameters of vancomycin for therapeutic regimens in neutropenic adult patients. Int J Biomed Comput 36:121-125

16. Cockcroft DW, Gault MH (1976) Prediction of creatinine clearance from serum creatinine. Nephron 16:31-41. https://doi.org/10.1159/ 000180580

17. Keizer RJ, Karlsson MO, Hooker A (2013) Modeling and simulation workbench for NONMEM: tutorial on Pirana, PsN, and Xpose. CPT Pharmacometrics Syst Pharmacol 2. https://doi.org/10.1038/ psp.2013.24

18. Dosne A-G, Bergstrand M, Harling K, Karlsson MO (2016) Improving the estimation of parameter uncertainty distributions in nonlinear mixed effects models using sampling importance resampling. J Pharmacokinet Pharmacodyn 43:583-596. https://doi.org/ 10.1007/s10928-016-9487-8
19. Bergstrand M, Hooker AC, Wallin JE, Karlsson MO (2011) Prediction-corrected visual predictive checks for diagnosing nonlinear mixed-effects models. AAPS J 13:143-151. https://doi.org/ 10.1208/s12248-011-9255-Z

20. Olofsen E, Dahan A (2014) Using Akaike's information theoretic criterion in mixed-effects modeling of pharmacokinetic data: a simulation study. F1000Research. https://doi.org/10.12688/ f1000research.2-71.v2

21. Staatz CE, Byrne C, Thomson AH (2006) Population pharmacokinetic modelling of gentamicin and vancomycin in patients with unstable renal function following cardiothoracic surgery. Br J Clin Pharmacol 61:164-176. https://doi.org/10.1111/j.1365-2125.2005. 02547.x

22. Grace E (2012) Altered vancomycin pharmacokinetics in obese and morbidly obese patients: what we have learned over the past 30 years. J Antimicrob Chemother 67:1305-1310. https://doi.org/10. 1093/jac/dks066

23. Holford NHG, Anderson BJ (2017) Allometric size: the scientific theory and extension to normal fat mass. Eur J Pharm Sci 109:S59 S64

24. Pea F, Furlanut M, Negri C, Pavan F, Crapis M, Cristini F, Viale P (2009) Prospectively validated dosing nomograms for maximizing the pharmacodynamics of vancomycin administered by continuous infusion in critically ill patients. Antimicrob Agents Chemother 53: 1863-1867. https://doi.org/10.1128/AAC.01149-08

25. Curth H-M, Pelc A, Kutting F, Steffen H-M (2015) Augmented renal vancomycin clearance in cancer patients: a case report and review of the literature. Oncol Res Treat 38:182-184. https://doi. org/10.1159/000377652

26. Campassi ML, Gonzalez MC, Masevicius FD, Vazquez AR, Moseinco M, Navarro NC, Previgliano L, Rubatto NP, Benites MH, Estenssoro E, Dubin A (2014) Augmented renal clearance in critically ill patients: incidence, associated factors and effects on vancomycin treatment. Rev Bras Ter Intensiva 26:13-20

27. Zokufa HZ, Solem LD, Rodvold KA, Crossley KB, Fischer JH, Rotschafer JC (1989) The influence of serum albumin and alpha 1 -acid glycoprotein on vancomycin protein binding in patients with burn injuries. J Burn Care Rehabil 10:425-428

Publisher's note Springer Nature remains neutral with regard to jurisdictional claims in published maps and institutional affiliations. 